\section{Circadiane Rhythmik}

C. Vidal ${ }^{1}$ und W.-R. Külpmann ${ }^{2}$

${ }^{1}$ Landeskriminalamt Niedersachsen, Dezernat 53 „Chemie“, Hannover, Deutschland

${ }^{2}$ Hannover, Deutschland

Synonym(e) Circadianer Rhythmus; Zirkadiane Rhythmik; Zirkadianer Rhythmus

Englischer Begriff circadian rhythm

Definition Änderung von Körperfunktionen in Abhängigkeit von der Tageszeit.

\begin{tabular}{|l|l|l|} 
& $\begin{array}{l}\text { Plasmakonzentration }_{\max } \\
\text { (Uhrzeit) }\end{array}$ & $\begin{array}{l}\text { Plasmakonzentration }_{\min } \\
\text { (Uhrzeit) }\end{array}$ \\
\hline Kortisol & $6-10$ & $0-4$ \\
\hline Kalium & $14-16$ & $23-1$ \\
\hline Melatonin & $0-6$ & $7-22$ \\
\hline STH & $21-23$ & $1-21$
\end{tabular}

Beschreibung Als Folge circadianer Rhythmik finden sich in Abhängigkeit von der Tageszeit bestimmte Substanzen in unterschiedlicher Konzentration in Blut oder Urin; Beispiele s. folgende Tabelle:

Aus diesem Grunde soll beachtet werden:

- Stets Dokumentation der Uhrzeit bei der Probennahme

- Blutentnahme möglichst immer zur selben Uhrzeit (z. B. 8:00 Uhr)

- Bei Referenzintervallermittlung Einbezug des Zeitpunkts der Probennahme

- Durchführung der quantitativen Bestimmung im Urin möglichst mit 24-Stunden-Sammelurin

\section{Literatur}

Wisser H (1995) Einflussgrößen und Störgrößen. In: Greiling H, Gressner AM (Hrsg) Lehrbuch der klinischen Chemie und Pathobiochemie, 3. Aufl. Schattauer Verlag, Stuttgart 